\title{
Comparison of LI-RADS 2018 and KLCA-NCC 2018 for noninvasive diagnosis of hepatocellular carcinoma using magnetic resonance imaging
}

Sunyoung Lee', Seung-seob Kim', Dong ryul Chang', Hyerim Kim², and Myeong-Jin Kim ${ }^{1}$

'Department of Radiology and Research Institute of Radiological Science, Severance Hospital, Yonsei University College of Medicine, Seoul; ${ }^{2 B i o s t a t i s t i c s ~ C o l l a b o r a t i o n ~ U n i t, ~ D e p a r t m e n t ~ o f ~ B i o m e d i c a l ~ S y s t e m s ~ I n f o r m a t i c s, ~ Y o n s e i ~ U n i v e r s i t y ~ C o l l e g e ~ o f ~ M e d i c i n e, ~ S e o u l, ~}$ Korea

ECA-MRI

\section{LI-RADS 2018 -LR-5}
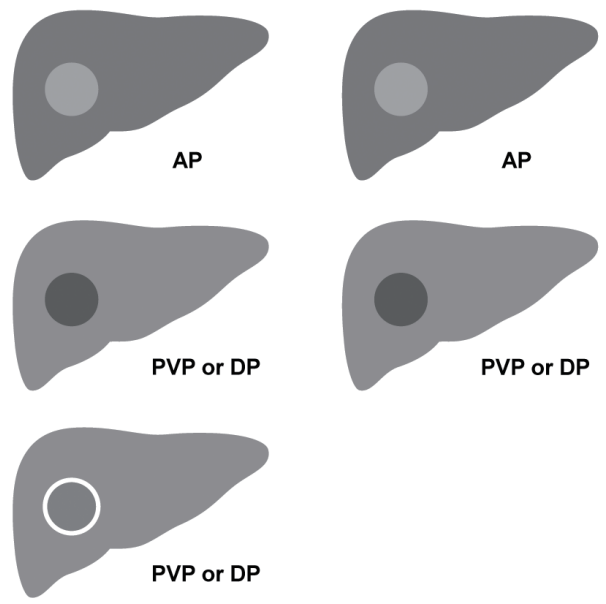

Sensitivity: LI-RADS $\fallingdotseq$ KLCA-NCC 2018

Specificity: LI-RADS $\fallingdotseq$ KLCA-NCC 2018
HBA-MRI

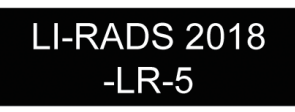

\section{KLCA-NCC 2018}

-definite HCC
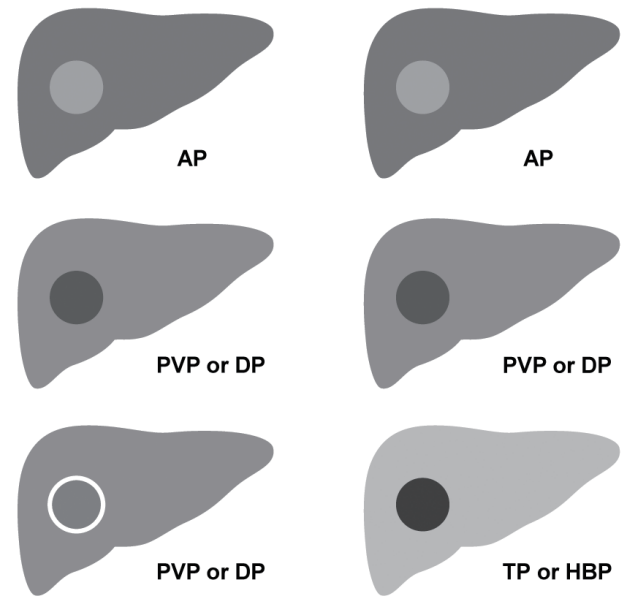

Sensitivity: LI-RADS < KLCA-NCC 2018

Specificity: LI-RADS $\fallingdotseq$ KLCA-NCC 2018

\section{Abbreviations:}

AP, arterial phase; CHCC-CCA, combined hepatocellular carcinomacholangiocarcinoma; DP, delayed phase; DWI, diffusion-weighted imaging; ECA, extracellular contrast agent; HBA, hepatobiliary agent; HBP, hepatobiliary phase; HCC, hepatocellular carcinoma; KLCA, Korean Liver Cancer Association; KLCSG, Korean Liver Cancer Study Group; LI-RADS, Liver Imaging Reporting and Data System; MRI, magnetic resonance imaging; NCC, National Cancer Center; PVP, portal venous phase; TP, transitional phase; US, ultrasound

\section{Corresponding author: Myeong-Jin Kim}

Department of Radiology and Research Institute of Radiological Science, Severance Hospital, Yonsei University College of Medicine, 50-1 Yonseiro, Seodaemun-gu, Seoul 03722, Korea

Tel: +82-2-2228-7400, Fax: +82-2-2227-8337

E-mail:kimnex@yuhs.ac

http://orcid.org/0000-0001-7949-5402 
Background/Aims: This study aimed to compare the diagnostic performances of Liver Imaging Reporting and Data System (LI-RADS) 2018 and Korean Liver Cancer Association-National Cancer Center (KLCA-NCC) 2018 criteria on magnetic resonance imaging (MRI) for the noninvasive diagnosis of hepatocellular carcinoma (HCC) in high-risk patients.

Methods: This retrospective study included 273 treatment-naïve patients (71 patients with extracellular contrast agent [ECA]-MRI and 202 patients with hepatobiliary agent [HBA]-MRI; 352 lesions including 263 HCCs) with high risk of HCC who underwent contrast-enhanced MRI between 2016 and 2017. Two readers evaluated all lesions according to the criteria of LI-RADS 2018 and KLCA-NCC 2018. The per-lesion diagnostic performances were compared using the generalized estimating equation method.

Results: On ECA-MRI, the sensitivity and specificity of LI-RADS 2018 and KLCA-NCC 2018 were not significantly different (LR-5 vs. definite HCC: $75.8 \%$ vs. $69.4 \%, P=0.095$ and $95.8 \%$ vs. $95.8 \%, P>0.999 ;$ LR- $5 / 4$ vs. definite/probable HCC: $87.1 \%$ vs.83.9\%, $P=0.313$ and $87.5 \%$ vs. $91.7 \%, P=0.307)$. On HBA-MRI, definite HCC of KLCA-NCC 2018 showed significantly higher sensitivity $(79.1 \%$ vs. $68.2 \%, P<0.001)$ than LR-5 of LI-RADS 2018 without a significant difference in specificity (93.9\% vs. $95.4 \%, P=0.314)$. Definite/probable HCC of KLCA-NCC 2018 had higher specificity $(92.3 \%$ vs. $80.0 \%, P=0.003)$ than LR$5 / 4$ of LI-RADS 2018. The sensitivity was lower for definite/probable HCC than for LR-5/4 without statistical significance ( $85.6 \%$ vs. $88.1 \%, P=0.057$ ).

Conclusions: On ECA-MRI, LI-RADS 2018 and KLCA-NCC 2018 showed comparable diagnostic performances. On HBA-MRI, definite HCC of KLCA-NCC 2018 provided better sensitivity than LR-5 category of LI-RADS 2018 without compromising the specificity, while definite/probable HCC of KLCA-NCC 2018 revealed higher specificity than LR-5/4 of LI-RADS 2018 for diagnosing HCC. (Clin Mol Hepatol 2020;26:340-351)

Keywords: Carcinoma, Hepatocellular; Diagnosis; Magnetic resonance imaging

\section{Study Highlights}

This study compared the LI-RADS 2018 and KLCA-NCC 2018 criteria on magnetic resonance imaging (MRI) for the noninvasive diagnosis of hepatocellular carcinoma (HCC). On extracellular contrast agent (ECA)-MRI, LI-RADS 2018 and KLCA-NCC 2018 show comparable diagnostic performances. On hepatobiliary agent (HBA)-MRI, definite HCC of KLCA-NCC 2018 provides better sensitivity than LR-5 of LI-RADS 2018 without a significant difference in the specificity.

\section{INTRODUCTION}

Hepatocellular carcinoma (HCC) is the most common primary liver cancer. It is the only malignancy of which the clinical practice for diagnosis is to use imaging without pathological confirmation in high-risk patients; thus, reliable noninvasive imaging criteria are crucial. ${ }^{2,3}$ To achieve consistent diagnosis of HCC, several scientific organizations have proposed imaging-based diagnostic criteria. The imaging criteria differ between geographic areas, reflecting differences in clinical environment and treatment strategies. ${ }^{4,5}$

The Liver Imaging Reporting and Data System (LI-RADS), endorsed by the American College of Radiology, is a comprehensive system to standardize the terminology, technique, interpretation, reporting, and data collection of liver imaging in patients at risk for HCC. ${ }^{6}$ LI-RADS is currently the most widely used noninvasive diagnostic criteria for HCC in radiological studies because it provides detailed definitions for major and ancillary imaging features of HCCS. LI-RADS categorizes an observation according to its probability of HCC (ranging from LR-1 to LR-5) or malignancy but not specific for HCC (LR-M), on the basis of major, ancillary, and LR-M imaging features. ${ }^{6}$ LI-RADS 2018 represents the fourth update and has been fully integrated into the American Association for the Study of Liver Disease 2018 HCC Practice Guidance. ${ }^{2}$ LIRADS was developed and modified predominantly based on data from Western countries and was designed to emphasize the specificity and positive predictive value over sensitivity for liver transplant eligibility. ${ }^{4,5}$

The Korean Liver Cancer Study Group (KLCSG)-National Cancer Center (NCC) HCC practice guidelines were first announced in 2003 and have been revised in 2009 and 2014. ${ }^{7-9}$ Since then, the Korean Liver Cancer Association (KLCA, formerly KLCSG)-NCC published KLCA-NCC 2018 practice guidelines for the management of $\mathrm{HCC} .{ }^{10}$ They provide diagnosis and treatment guideline specific to the Asian who manifest different clinical behaviors of 
HCC compared to Western population, especially in Korean. ${ }^{7-10}$ The KLCA-NCC 2018 practice guidelines for the imaging diagnosis of HCC has been revised into a non-binary decision algorithm that covers the whole spectrum of observations, similar to LI-RADS. ${ }^{10}$ The KLCA-NCC 2018 guidelines categorize an observation into indeterminate, probable HCC, and definite HCC after excluding benign lesions such as cyst or hemangiomas based on bright $\mathrm{T} 2$ signal intensity and other malignancies based on targetoid appearance. ${ }^{10,11}$ KLCA-NCC 2018 was designed to favor high sensitivity for the detection of early HCC and early treatment with locoregional therapies. ${ }^{4,5}$

However, data to support these updated versions of the imaging criteria are insufficient; moreover, limited data are available to compare the LI-RADS 2018 and KLCA-NCC 2018 criteria for the noninvasive diagnosis of HCC. Therefore, this study aimed to compare the diagnostic performances of LI-RADS 2018 and KLCANCC 2018 criteria on magnetic resonance imaging (MRI) for diagnosing HCC in high-risk patients.

\section{MATERIALS AND METHODS}

This retrospective study was approved by the Institutional Review Board of Severance Hospital (IRB number 4-2019-1127) and the requirement for informed consent was waived.

\section{Patients}

We searched our institution's databases for a clinical cohort under HCC surveillance and identified 2,576 patients who underwent MRI for further diagnostic workup between January 2016 and December 2017. The inclusion criteria were as follows: 1) age of 18 years or older; 2) patients at high risk for HCC with cirrhosis or chronic hepatitis $\mathrm{B}$; 3 ) presence of at least one and up to five hepatic lesions (each $\geq 1 \mathrm{~cm}$ ) on MRl; and 4) no previous treatment for hepatic lesions. The exclusion criteria were as follows: 1) patients with cirrhosis due to congenital hepatic fibrosis and vascular disorders such as hereditary hemorrhagic telangiectasia, Budd-Chiari syndrome, chronic portal vein occlusion, cardiac congestion, or diffuse nodular regenerative hyperplasia $(n=17)$; and 2) patients with insufficient final diagnosis such as unknown final diagnosis of malignancy as a result of immediate locoregional therapy or insufficient follow-up ( $<2$ years) for benign lesions to determine size stability $(n=579)$. We did not include observations such as hepatic cysts, perfusion alteration, hepatic fat deposition or sparing, hypertrophic pseudomass, confluent fibrosis, and focal scar. However, we include hemangiomas in our study. A radiologist (S.K., with 9 years of experience in abdominal radiology) who was not involved in the image analysis for this study finalized the study population and selected hepatic lesions.

\section{MRI examination}

MRI was performed using 3.0-T systems (Magnetom Trio Tim, Siemens Healthineers, Erlangen, Germany; Intera Achieva, Ingenia, or Ingenia CX, Philips Healthcare, Best, the Netherlands; and Discovery MR 750w, GE Healthcare, Waukesha, WI, USA). The protocol included acquisition of dual-echo T1-weighted gradientecho images (in-phase and opposed-phase), T1-weighted 3-dimensional gradient-echo images with dynamic contrast enhancement, navigator-triggered single- or multi-shot T2-weighted images, and diffusion-weighted images at $b$-values of 0 or 50 , 400 , and $800 \mathrm{~s} / \mathrm{mm}^{2}$. Dynamic T1-weighted imaging was performed before and after administering one of the two extracellular contrast agents (ECAs) (gadoterate meglumine, Dotarem, Guerbet SA, Aulnay-sous-Bois, France; or gadobutrol, Gadovist, Bayer Pharma AG, Berlin, Germany) or a hepatobiliary agent (HBA) (gadoxetate disodium, Primovist, Bayer Pharma AG): a bolus injection of $0.2 \mathrm{~mL} / \mathrm{kg}$ of gadoterate meglumine $(n=67)$ at a rate of $2.0 \mathrm{~mL} / \mathrm{s}$ or $0.1 \mathrm{~mL} / \mathrm{kg}$ of gadobutrol $(n=4)$ at a rate of $1.0 \mathrm{~mL} / \mathrm{s}$, or $0.1 \mathrm{~mL} / \mathrm{kg}$ gadoxetate disodium ( $\mathrm{n}=202)$ at a rate of $1.0 \mathrm{~mL} / \mathrm{s}$, followed by a $20-\mathrm{mL}$ saline flush using a power injector. The choice of MRI contrast agents (ECA or HBA) was made at the discretion of the physicians. The physicians were fully informed by radiologists regarding the advantages and disadvantages of each contrast material at a multidisciplinary conference in our institution, such as the potential differences in terms of degree of arterial phase (AP) enhancement and relative frequency of artifacts between the two contrast materials, potential benefits of hepatobiliary phase (HBP) images, and cost of contrast materials. AP scanning was initiated using the test-bolus or bolus-tracking technique. Thereafter, portal venous phase (PVP) and delayed phase (DP) images were obtained by ECA-MRI, while PVP, transitional phase (TP), and 20-minute HBP images were obtained by HBA-MRI. The detailed parameters of the MRI sequences are presented in Supplementary Table 1.

\section{Lesion registry}

A radiologist (S.K., with 9 years of experience in abdominal ra- 
diology) who was not involved in the image analysis bookmarked individual lesions, reported the lesion size and location, and provided a list for further review. The prior computed tomography or MRI examination was used to assess threshold or subthreshold growth, and ultrasound (US) was used to assess US visibility as a discrete nodule when available.

\section{Image analysis}

Two board-certified abdominal radiologists (M.J.K. and S.L. with 27 and 8 years of experience in liver imaging, respectively) who did not participate in the patient and lesion selection reviewed all images. They were blinded to the final diagnosis of each lesion but were informed that the study population consisted of patients at high risk for HCC. Both LI-RADS 2018 and KLCANCC 2018 criteria were independently applied to each lesion. After independent categorization, the inter-reader agreement was evaluated. Discrepancies between the readers were resolved by consensus. Consensus data were used for the main image analysis results.

\section{LI-RADS 2018}

According to LI-RADS 2018, the readers reviewed the presence or absence of major, ancillary, and LR-M imaging features (Supplementary Table 2). ${ }^{6}$ Each lesion was assigned to one of the following categories: LR-1 for definitely benign, LR-2 for probably benign, LR-3 for intermediate probability of malignancy, LR-4 for probably HCC, LR-5 for definitely HCC, LR-M for probably or definitely malignant but not specific for HCC, and LR-TIV for definite tumor in vein. ${ }^{6}$ The LI-RADS 2018 category could be adjusted by applying ancillary features and then tie-breaking rules, if needed. ${ }^{6}$

\section{KLCA-NCC 2018}

According to KLCA-NCC 2018, the readers assessed the presence or absence of major and ancillary imaging features and targetoid appearance (Supplementary Table 3).10,11 Each lesion was categorized as definite HCC, probable HCC, indeterminate, benign, and targetoid appearance. Definite HCC was defined as AP hyperenhancement with washout in the PVP, DP, or HBP. ${ }^{10,11}$ In a lesion with some but not all of the major imaging features of HCC, probable HCC was assigned only when the lesion fulfilled at least one item from each of the following two categories of ancillary imaging features: favoring malignancy in general, and favoring HCC in particular. ${ }^{10,11}$ The diagnostic criteria for HCC should be applied in a stepwise manner after excluding marked $\mathrm{T} 2$ hyperinten- sity or targetoid appearance. ${ }^{10,11}$ When the imaging diagnosis was inconclusive, a lesion was defined as indeterminate. ${ }^{10,11}$ Although the KLCA-NCC 2018 guidelines do not clearly define benign and other malignancy categories, we added benign lesions and targetoid appearance to the system in order to match the classification with the LI-RADS 2018. A lesion that fulfilled at least one of the ancillary features favoring benignity according to KLCA-NCC $2018^{10,11}$ was classified as a benign lesion. Targetoid appearance was evaluated on diffusion-weighted imaging (DWI) or contrastenhanced sequences.

\section{Comparison of LI-RADS 2018 and KLCA-NCC 2018}

Definite HCC of KLCA-NCC 2018 corresponds to LR- 5 of LIRADS 2018, and probable HCC of KLCA-NCC 2018 corresponds to LR-4 of LI-RADS 2018. For the noninvasive diagnosis of HCC, definite HCC of KLCA-NCC 2018 was compared with LR-5 of LIRADS 2018, and definite or probable HCC (definite/probable HCC) of KLCA-NCC 2018 was compared with LR-5 or LR-4 (LR-5/4) of LI-RADS 2018.

\section{Reference standards}

The diagnoses of HCCs and non-HCC malignancies were based on pathology including surgical resection $(n=285)$ or explant for transplantation ( $n=15)$. Benign diagnoses were obtained by pathology $(n=14)$ or typical imaging features and stability at imaging for at least 2 years $(n=38)$. The mean interval between the MRI and pathological diagnosis was 20.2 days (range, $0-90$ days).

\section{Statistical analysis}

The baseline characteristics of patients and lesions were compared between the ECA-MRI and HBA-MRI groups using the Fisher's exact test for categorical variables and the two-sample $t$ test or Mann-Whitney $U$ test for continuous variables. The per-lesion diagnostic performances of LI-RADS 2018 and KLCA-NCC 2018 were compared by using the generalized estimating equation method. The inter-reader agreement was evaluated using the Cohen $\mathrm{k}$ coefficient. The $\mathrm{k}$ values were interpreted as follows: poor, 0.00-0.20; fair, 0.21-0.40; moderate, 0.41-0.60; good, $0.61-$ 0.80; and excellent, 0.81-1.00. Statistical analyses were performed using SAS software (version 9.4; SAS Inc., Cary, NC, USA). A $P$-value of $<0.05$ was considered statistically significant. 


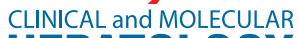

\section{HEPATOLOGY}

Volume_26 Number_3 July 2020

\section{RESULTS}

\section{Characteristics of patients and lesions}

A total of 273 patients (mean age, 57.3 years; 188 men and 85 women) with 352 lesions were selected for the final statistical analysis (Fig. 1). The characteristics of the patients and lesions are presented in Table 1. The study consisted of 71 patients with 86 lesions who underwent ECA-MRI, and 202 patients with 266 lesions who underwent HBA-MRI. No statistically significant differences in sex, age, presence of liver cirrhosis, lesion size, proportions of categories according to LI-RADS 2018 and KLCA-NCC 2018, and final diagnosis were found between ECA-MRI and HBA-MRI groups. Hepatitis B (89.8\%) was the predominant etiology of liver disease. Two hundred eighteen (79.9\%) patients had one lesion; 37 (13.6\%) had two lesions; and 18 (6.5\%) had three or more lesions. The 352 lesions included $263(74.7 \%)$ HCCs, 37 (10.5\%) non-HCC malignancies, and 52 (14.8\%) benign lesions.

\section{LR-5 of LI-RADS 2018 versus definite HCC of KLCA- NCC 2018}

The per-lesion diagnostic performances for LR-5 of LI-RADS 2018 and definite HCC of KLCA-NCC 2018 are demonstrated in Table 2.

On ECA-MRI, the sensitivity and specificity of LR-5 of LI-RADS
2018 and definite HCC of KLCA-NCC 2018 were not significantly different $(75.8 \%$ vs. $69.4 \%, P=0.095$ and $95.8 \%$ vs. $95.8 \%$, $P>0.999)$ for the entire cohort. In both subgroups according to the size of lesions (10-19 mm and $\geq 20 \mathrm{~mm}$ ), there were no significantly differences in the sensitivities and specificities between LR-5 and definite HCC (all, $P>0.05$ ).

On HBA-MRI, the sensitivity was significantly higher for definite HCC of KLCA-NCC 2018 (79.1\% vs. 68.2\%, $P<0.001)$ than for LR-5 of LI-RADS 2018 without a significant difference in specificity $(93.9 \%$ vs. $95.4 \%, P=0.314)$ for all lesions. When lesions were stratified according to the size (10-19 mm and $\geq 20 \mathrm{~mm}$ ), the sensitivities of definite $\mathrm{HCC}$ were significantly higher than those of LR-5 (10-19 mm: $75.8 \%$ vs. $51.6 \%, P<0.001$; and $\geq 20 \mathrm{~mm}$ : $80.6 \%$ vs. $75.5 \%, P=0.017)$, but the specificities were not significantly different (all, $P>0.05$ ), similar to the results obtained for all lesions (Figs. 2, 3).

There were 27 lesions with threshold growth (24 HCCs and three non-HCC malignancies) according to LI-RADS 2018. By using only threshold growth (one of the major imaging features of LI-RADS 2018) with AP hyperenhancement, two additional HCCS were diagnosed as LR-5.

\section{LR-5/4 of LI-RADS 2018 versus definite/probable HCC of KLCA-NCC 2018}

The per-lesion diagnostic performances of LR-5/4 of LI-RADS

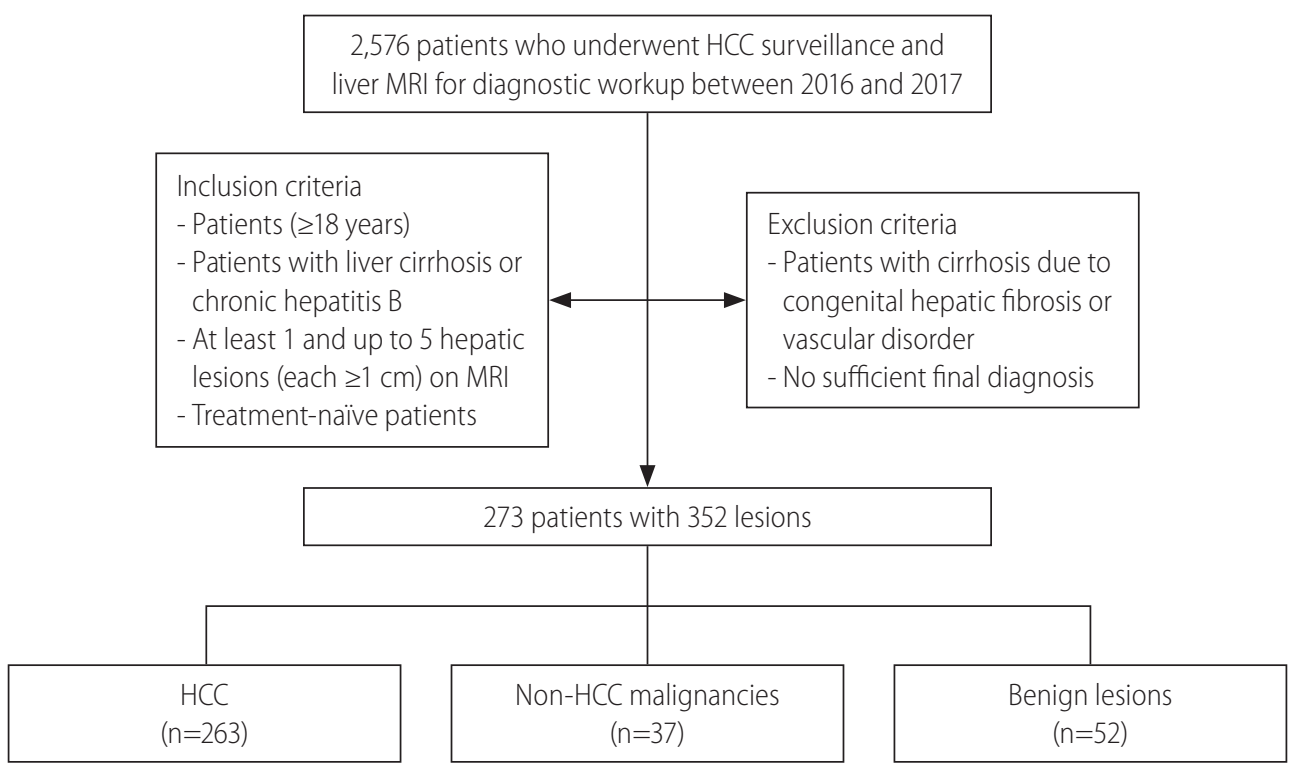

Figure 1. Flowchart of study population. HCC, hepatocellular carcinoma; MRI, magnetic resonance imaging. 
Table 1. Characteristics of 273 patients and 352 lesions

\begin{tabular}{|c|c|c|c|c|}
\hline Characteristic & Total & ECA-MRI & HBA-MRI & $P$-value \\
\hline Patients & 273 & 71 & 202 & \\
\hline Sex, male & $188(68.9)$ & $46(64.8)$ & $142(70.3)$ & 0.456 \\
\hline Age (years) ${ }^{*}$ & $57.3 \pm 9.5$ & $57.4 \pm 9.9$ & $57.3 \pm 9.4$ & 0.950 \\
\hline Etiology & & & & 0.022 \\
\hline Hepatitis B & $245(89.8)$ & $60(84.5)$ & $185(91.6)$ & \\
\hline Hepatitis C & $11(4.0)$ & $7(9.9)$ & $4(2.0)$ & \\
\hline Alcohol & $17(6.2)$ & $4(5.6)$ & $13(6.4)$ & \\
\hline Liver cirrhosis & $150(54.9)$ & $39(54.9)$ & $111(55.0)$ & 0.998 \\
\hline Lesions & 352 & 86 & 266 & \\
\hline Size $(m m)^{\dagger}$ & $24(15-34)$ & $25(19-32)$ & $24(15-35)$ & 0.416 \\
\hline Size subgroup & & & & 0.307 \\
\hline $10-19 \mathrm{~mm}$ & $132(37.5)$ & $28(32.6)$ & $104(39.1)$ & \\
\hline$\geq 20 \mathrm{~mm}$ & $220(62.5)$ & $58(67.4)$ & $162(60.9)$ & \\
\hline Categorization according to LI-RADS 2018 & & & & 0.111 \\
\hline LR-2 & $30(8.5)$ & $7(8.1)$ & $23(8.7)$ & \\
\hline LR-3 & $14(4.0)$ & $7(8.1)$ & $7(2.6)$ & \\
\hline$L R-4$ & $59(16.8)$ & $9(10.5)$ & $50(18.8)$ & \\
\hline LR-5 & $188(53.4)$ & $48(55.8)$ & $140(52.6)$ & \\
\hline LR-M & $59(16.8)$ & $14(16.3)$ & $45(16.9)$ & \\
\hline LR-TIV & $2(0.5)$ & $1(1.2)$ & $1(0.4)$ & \\
\hline Categorization according to KLCA-NCC 2018 & & & & 0.135 \\
\hline Benign & $31(8.8)$ & $11(12.8)$ & $20(7.5)$ & \\
\hline Indeterminate & $31(8.8)$ & $7(8.1)$ & $24(9.0)$ & \\
\hline Probable HCC & $24(6.8)$ & $10(11.6)$ & $14(5.3)$ & \\
\hline Definite HCC & $207(58.8)$ & $44(51.2)$ & $163(61.3)$ & \\
\hline Targetoid appearance & $59(16.8)$ & $14(16.3)$ & 45 (16.9) & \\
\hline Final diagnosis & & & & 0.051 \\
\hline $\mathrm{HCC}$ & $263(74.7)$ & $62(72.1)$ & $201(75.6)$ & \\
\hline Non-HCC malignancy & $37(10.5)$ & $9(10.5)$ & $28(10.5)$ & \\
\hline CHCC-CCA & $23(6.6)$ & $5(5.8)$ & $18(6.8)$ & \\
\hline Intrahepatic cholangiocarcinoma & $9(2.5)$ & $3(3.5)$ & $6(2.2)$ & \\
\hline Hepatoblastoma & $1(0.3)$ & $1(1.2)$ & $0(0.0)$ & \\
\hline Metastasis & $4(1.1)$ & $0(0.0)$ & $4(1.5)$ & \\
\hline Benign lesion & $52(14.8)$ & $15(17.4)$ & $37(13.9)$ & \\
\hline Hemangioma & $14(4.0)$ & $4(4.6)$ & $10(3.8)$ & \\
\hline Focal nodular hyperplasia-like nodule & $8(2.3)$ & $6(7.0)$ & $2(0.7)$ & \\
\hline Regenerative nodule & $17(4.8)$ & $3(3.5)$ & $14(5.3)$ & \\
\hline Dysplastic nodule & $13(3.7)$ & $2(2.3)$ & $11(4.1)$ & \\
\hline
\end{tabular}

Values are presented as mean \pm standard deviation, median (interquartile), or number (\%).

ECA, extracellular contrast agent; MRI, magnetic resonance imaging; HBA, hepatobiliary agent; LI-RADS, Liver Imaging Reporting and Data System; KLCA, Korean Liver Cancer Association; HCC, hepatocellular carcinoma; CHCC-CCA, combined hepatocellular carcinoma-cholangiocarcinoma.

*Compared by using the two-sample $t$ test.

${ }^{\dagger}$ Compared by using Mann-Whitney $U$ test. 


\section{CLINCAL and MOLECULAR}

Volume_26 Number_3 July 2020

Table 2. Diagnostic performances of LR-5 of LI-RADS 2018 and definite HCC of KLCA-NCC 2018 for diagnosing HCC on ECA-MRI and HBA-MRI

\begin{tabular}{|c|c|c|c|}
\hline & $\begin{array}{l}\text { LR-5 of LI-RADS } 2018 \\
\qquad(95 \% \mathrm{Cl})\end{array}$ & $\begin{array}{l}\text { Definite HCC of KLCA-NCC } 2018 \\
(95 \% \mathrm{Cl})\end{array}$ & $P$-value* \\
\hline \multicolumn{4}{|c|}{ ECA-MRI all $(n=86)$} \\
\hline Sensitivity & $75.8(65.2-86.5)$ & $69.4(57.9-80.8)$ & 0.095 \\
\hline Specificity & $95.8(87.8-100.0)$ & $95.8(87.8-100.0)$ & $>0.999$ \\
\hline \multicolumn{4}{|c|}{ ECA-MRI 10-19 mm $(n=28)$} \\
\hline Sensitivity & $58.8(35.4-82.2)$ & $58.8(35.4-82.2)$ & $>0.999$ \\
\hline Specificity & $100.0(100.0-100.0)$ & $100.0(100.0-100.0)$ & $>0.999$ \\
\hline \multicolumn{4}{|c|}{$\mathrm{ECA}-\mathrm{MRI} \geq 20 \mathrm{~mm}(\mathrm{n}=58)$} \\
\hline Sensitivity & $82.2(71.1-93.4)$ & $73.3(60.4-86.3)$ & 0.092 \\
\hline Specificity & $92.3(77.8-100.0)$ & $92.3(77.8-100.0)$ & 0.109 \\
\hline \multicolumn{4}{|c|}{ HBA-MRI all $(n=266)$} \\
\hline Sensitivity & $68.2(61.7-74.6)$ & $79.1(73.5-84.7)$ & $<0.001$ \\
\hline Specificity & $95.4(90.3-100.0)$ & $93.9(88.0-99.7)$ & 0.314 \\
\hline \multicolumn{4}{|c|}{ HBA-MRI 10-19 mm $(n=104)$} \\
\hline Sensitivity & $51.6(39.2-64.1)$ & $75.8(65.2-86.5)$ & $<0.001$ \\
\hline Specificity & $97.6(93.0-100.0)$ & $95.2(88.8-100.0)$ & 0.312 \\
\hline \multicolumn{4}{|c|}{ HBA-MRI $\geq 20 \mathrm{~mm}(\mathrm{n}=162)$} \\
\hline Sensitivity & $75.5(68.4-82.7)$ & $80.6(74.0-87.2)$ & 0.017 \\
\hline Specificity & $91.3(79.8-100.0)$ & $91.3(79.8-100.0)$ & $>0.999$ \\
\hline
\end{tabular}

LI-RADS, Liver Imaging Reporting and Data System; HCC, hepatocellular carcinoma; KLCA-NCC, Korean Liver Cancer Association-National Cancer Center; ECA, extracellular contrast agent; MRI, magnetic resonance imaging; HBA, hepatobiliary agent; $\mathrm{Cl}$, confidence interval.

* $P$-values between LR-5 of LI-RADS 2018 and definite HCC of KLCA-NCC 2018 by using the generalized estimating equation method.

2018 and definite/probable HCC of KLCA-NCC 2018 are summarized in Table 3.

On ECA-MRI, no significant differences in the sensitivity and specificity were observed between LR-5/4 of LI-RADS 2018 and definite/probable HCC of KLCA-NCC 2018 (87.1\% vs. 83.9\%, $P=0.313$ and $87.5 \%$ vs. $91.7 \%, P=0.307$ ) for the entire cohort. When lesions were stratified according to the size (10-19 $\mathrm{mm}$ and $\geq 20 \mathrm{~mm}$ ), the sensitivities and specificities of LR-5/4 and definite/ probable HCC were not significantly different for both subgroups (all, $P>0.05$ ).

On HBA-MRI, definite/probable HCC of KLCA-NCC 2018 showed significantly higher specificity $(92.3 \%$ vs. $80.0 \%$, $P=0.003)$ than LR-5/4 of LI-RADS 2018 for diagnosing HCC without a statistically significant difference in sensitivity $(85.6 \%$ vs. $88.1 \%, P=0.057)$ for all lesions. The specificity of definite/probable HCC for 10-19 mm-sized lesions was significantly higher than that of $L R-5 / 4(92.9 \%$ vs. $73.8 \%, P=0.002)$, but the sensitivities for 10-19 mm-sized lesions and $\geq 20 \mathrm{~mm}$-sized lesions were not significantly different (all, $P>0.05$ ).

\section{False-positive diagnoses for HCC}

There were four false-positive diagnoses in LR-5 of LI-RADS 2018 and five false-positive diagnoses in definite HCC of KLCANCC 2018. All false-positive results were combined hepatocellular carcinoma-cholangiocarcinoma (cHCC-CCA) (Fig. 4, Supplementary Table 4).

In LR-5/4 of LI-RADS 2018, the false-positive results were eight non-HCC malignancies (six HCC-CCAs, one hepatoblastoma, and one metastasis) and eight benign lesions (two focal nodular hyperplasia-like nodules and six dysplastic nodules). In definite/ probable HCC of KLCA-NCC 2018, the false-positive diagnoses were six CHCC-CCAs and one hepatoblastoma (Supplementary Table 5).

\section{Subgroup analysis in patients with hepatitis B}

Supplementary Table 6 and 7 present the subgroup analysis results in patients with hepatitis $B$. The results of patients with hep- 

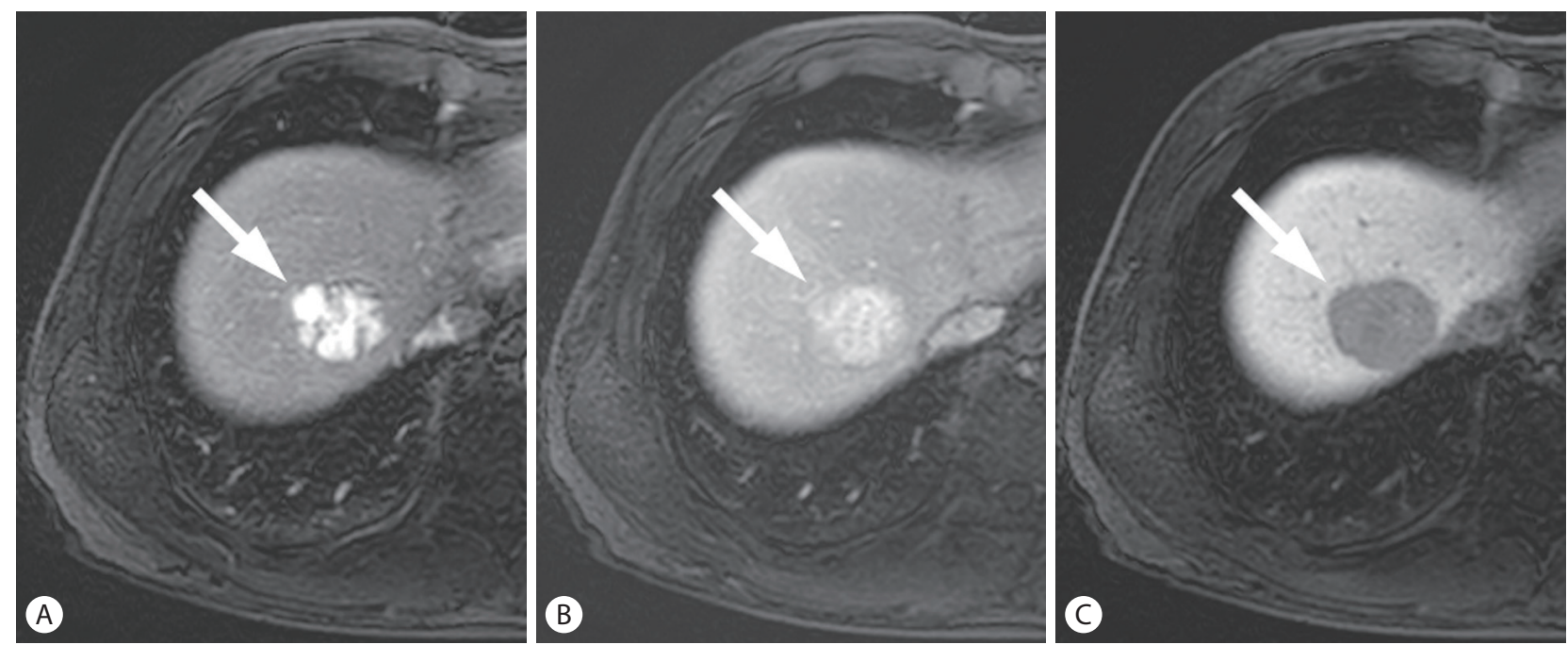

Figure 2. HCC in a 53-year-old man with chronic hepatitis B. On the arterial (A), portal venous (B), and hepatobiliary phase (C) images, after administration of hepatobiliary agent, a 39-mm liver mass (arrows) showed arterial phase hyperenhancement without washout in the portal venous phase, while showing hypointensity in the hepatobiliary phase. The mass was categorized as LR-4 by LI-RADS 2018, but classified as definite HCC by KLCA-NCC 2018. HCC, hepatocellular carcinoma; LI-RADS, Liver Imaging Reporting and Data System; KLCA-NCC, Korean Liver Cancer Association-National Cancer Center.
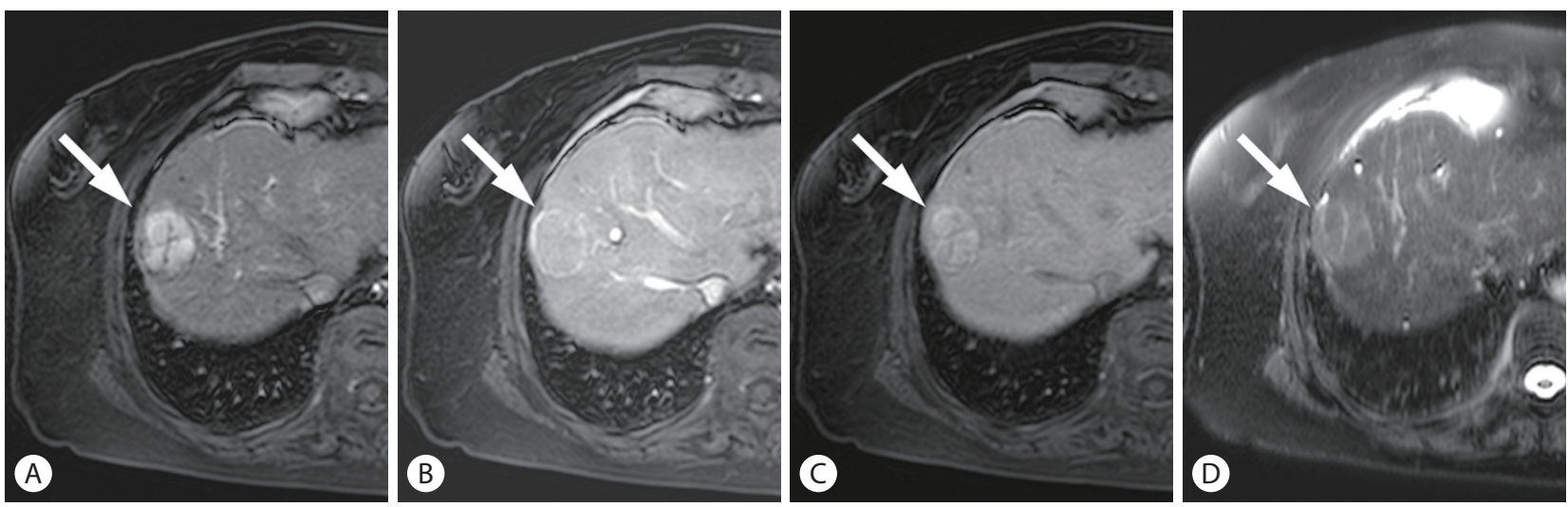

Figure 3. HCC in a 60-year-old woman with cirrhosis related to hepatitis B. On the arterial (A), portal venous (B), and hepatobiliary phase (C) images after administration of hepatobiliary agent, a 36-mm liver mass (arrows) showed arterial phase hyperenhancement without washout but with enhancing capsule in the portal venous phase, and hyperintensity in the hepatobiliary phase. On T2-weighted image (D), the mass demonstrated mild-moderate T2 hyperintensity. The mass was categorized as LR-5 by LI-RAD 2018, but classified as probable HCC by KLCA-NCC 2018. HCC, hepatocellular carcinoma; LI-RADS, Liver Imaging Reporting and Data System; KLCA-NCC, Korean Liver Cancer Association-National Cancer Center.

atitis B were similar and in line with those of the entire cohort.

On ECA-MRI, the sensitivity and specificity of LI-RADS 2018 and KLCA-NCC 2018 were not significantly different (LR-5 vs. definite HCC: $75.0 \%$ vs. $67.9 \%, P=0.094$ and $93.3 \%$ vs. $93.3 \%$, $P>0.999$; LR-5/4 vs. definite/probable HCC: $87.5 \%$ vs. $83.9 \%$, $P=0.313$ and $86.7 \%$ vs. $86.7 \%, P>0.999$ ).

On HBA-MRI, definite HCC of KLCA-NCC 2018 showed significantly higher sensitivity $(79.1 \%$ vs. $68.5 \%, P<0.001)$ than LR-5 of LI-RADS 2018 without a significant difference in specificity
(94.9\% vs. $96.6 \%, P=0.313$ ). Definite/probable HCC of KLCANCC 2018 had higher specificity (93.2\% vs. 81.4\%, $P=0.005)$ than LR-5/4 of LI-RADS 2018. The sensitivity was lower for definite/probable HCC than for LR-5/4 without statistical significance ( $85.0 \%$ vs. $87.7 \%, P=0.056$ ).

\section{Inter-reader agreement}

The inter-reader agreement for the categorization of lesions ac- 


\section{CLINCAL and MOLECULAR}

Volume_26 Number_3 July 2020

Table 3. Diagnostic performances of LR-5/4 of LI-RADS 2018 and definite/probable HCC of KLCA-NCC 2018 for diagnosing HCC on ECA-MRI and HBAMRI

\begin{tabular}{|c|c|c|c|}
\hline & $\begin{array}{l}\text { LR-5/4 of LI-RADS } 2018 \\
(95 \% \mathrm{Cl})\end{array}$ & $\begin{array}{l}\text { Definite/probable HCC of } \\
\text { KLCA-NCC } 2018(95 \% \text { CI) }\end{array}$ & $P$-value* \\
\hline \multicolumn{4}{|c|}{ ECA-MRI all $(n=86)$} \\
\hline Sensitivity & $87.1(78.8-95.4)$ & $83.9(74.7-93.0)$ & 0.313 \\
\hline Specificity & $87.5(74.3-100.0)$ & $91.7(80.6-100.0)$ & 0.307 \\
\hline \multicolumn{4}{|c|}{ ECA-MRI 10-19 mm (n=28) } \\
\hline Sensitivity & $94.1(82.9-100.0)$ & $76.5(56.3-96.6)$ & 0.056 \\
\hline Specificity & $100.0(100.0-100.0)$ & $100.0(100.0-100.0)$ & $>0.999$ \\
\hline \multicolumn{4}{|c|}{$\mathrm{ECA}-\mathrm{MRI} \geq 20 \mathrm{~mm}(\mathrm{n}=58)$} \\
\hline Sensitivity & $84.4(73.9-95.0)$ & $86.7(76.7-96.6)$ & 0.312 \\
\hline Specificity & $76.9(54.0-99.8)$ & $84.6(65.0-100.0)$ & 0.298 \\
\hline \multicolumn{4}{|c|}{ HBA-MRI all $(n=266)$} \\
\hline Sensitivity & $88.1(83.6-92.5)$ & $85.6(80.7-90.4)$ & 0.057 \\
\hline Specificity & $80.0(70.3-89.7)$ & $92.3(85.8-98.8)$ & 0.003 \\
\hline \multicolumn{4}{|c|}{ HBA-MRI 10-19 mm $(\mathrm{n}=104)$} \\
\hline Sensitivity & $87.1(78.8-95.4)$ & $82.3(72.8-91.8)$ & 0.076 \\
\hline Specificity & $73.8(60.5-87.1)$ & $92.9(85.1-100.0)$ & 0.002 \\
\hline \multicolumn{4}{|c|}{ HBA-MRI $\geq 20 \mathrm{~mm}(\mathrm{n}=162)$} \\
\hline Sensitivity & $88.5(83.2-93.8)$ & $87.1(81.5-92.6)$ & 0.316 \\
\hline Specificity & $91.3(79.8-100.0)$ & $91.3(79.8-100.0)$ & $>0.999$ \\
\hline
\end{tabular}

LI-RADS, Liver Imaging Reporting and Data System; HCC, hepatocellular carcinoma; KLCA-NCC, Korean Liver Cancer Association-National Cancer Center; ECA extracellular contrast agent; MRI, magnetic resonance imaging; HBA, hepatobiliary agent; $\mathrm{Cl}$, confidence interval.

* $P$-values between LR-5/4 of LI-RADS 2018 and definite/probable HCC of KLCA-NCC 2018 by using the generalized estimating equation method.
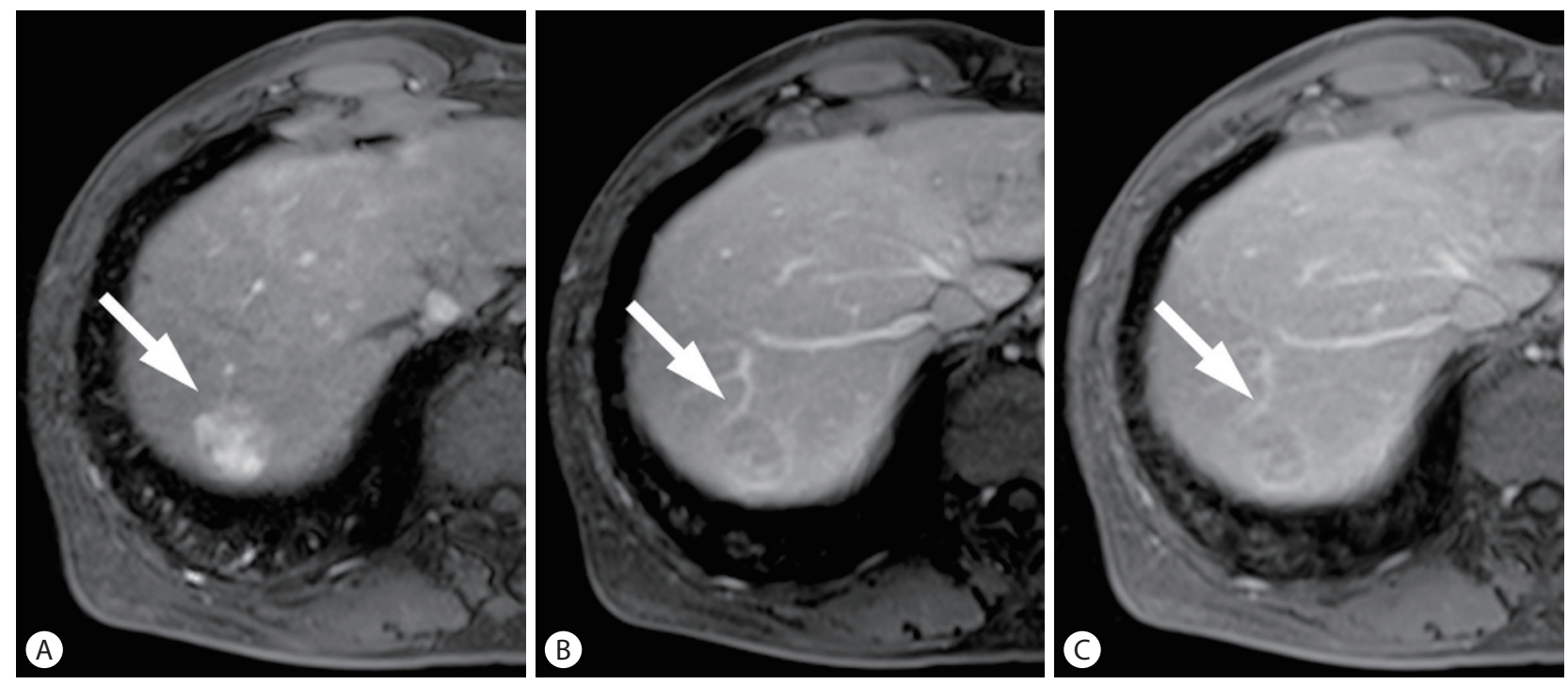

Figure 4. CHCC-CCA in a 62-year-old man with chronic hepatitis B. On the arterial (A), portal venous (B), and delayed phase (C) images, after administration of extracellular contrast agent, a 26-mm liver mass (arrows) showed arterial phase hyperenhancement with washout and enhancing capsule in the portal venous and delayed phases. The mass was categorized as LR-5 by LI-RADS 2018, and classified as definite HCC by KLCA-NCC 2018. However, it was diagnosed as CHCC-CCA by surgical resection. CHCC-CCA, combined hepatocellular carcinoma-cholangiocarcinoma; LI-RADS, Liver Imaging Reporting and Data System; KLCA-NCC, Korean Liver Cancer Association-National Cancer Center. 
cording to LI-RADS 2018 and KLCA-NCC 2018 was excellent $(K=0.90-0.97)$. The detailed results of inter-reader agreement are provided in Supplementary Table 8.

\section{DISCUSSION}

Our study showed that LI-RADS 2018 and KLCA-NCC 2018 had comparable diagnostic performances on ECA-MRI. On HBA-MRI, definite HCC of KLCA-NCC 2018 showed better sensitivity without a significant change in specificity, compared to LR-5 of LIRADS 2018. Definite/probable HCC of KLCA-NCC 2018 provided higher specificity than LR-5/4 of LI-RADS 2018 when using HBAMRI.

Our study indicates that KLCA-NCC 2018 may provide better diagnostic performance than LI-RADS 2018 to diagnose HCC with HBA-MRI, but not with ECA-MRI. The discrepancy may be attributed to the different definitions of washout appearance between the two systems. LI-RADS 2018 states that washout should be determined in the PVP only for HBA-MRI, ${ }^{6}$ whereas KLCA-NCC 2018 defines washout not only in the PVP but also in the TP or HBP. ${ }^{10,11}$ In our study, on HBA-MRI, the use of washout appearance defined by KLCA-NCC 2018 enabled the diagnosis of 23 additional HCC lesions compared to that defined by LI-RADS 2018, which resulted in increased sensitivity for definite HCC of KLCANCC 2018. This result is consistent with the results of recent studies that reported improved sensitivity by using HBP hypointensity as an alternative to washout on HBA-MRI. ${ }^{12-14}$ Nevertheless, definite HCC of KLCA-NCC 2018 did not significantly reduce its specificity compared with LR-5 of LI-RADS 2018. The diagnostic criteria for HCC according to KLCA-NCC 2018 may prevent significant loss in specificity by excluding hemangiomas or other malignancies, based on ancillary features favoring benignity such as marked T2 hyperintensity or targetoid appearance on DWI or contrast-enhanced sequences prior to the application of major imaging features. The use of a sequential algorithm with stepwise consideration in KLCA-NCC 2018 as in LI-RADS 2018 seems to help maintain a high specificity for the diagnosis of HCC. $5,10,11,15,16$

LI-RADS 2018 and KLCA-NCC 2018 are unique compared to other noninvasive imaging criteria in that they adapt non-binary decision algorithms and incorporate ancillary imaging features to modulate the likelihood of HCC. They allow categories for probably HCC (designated as LR-4 of LI-RADS 2018 and probable HCC of KLCA-NCC 2018) with different combinations of major and ancillary imaging features. Observations that do not meet stringent
LR-5 criteria of LI-RADS or lesions with some but not all of the major imaging features of KLCA-NCC 2018 criteria can be appropriately categorized as LR-4 of LI-RADS 2018 or probable HCC of KLCA-NCC 2018. While an observation can be upgraded from LR-3 to LR-4 with any of the ancillary features favoring malignancy in LI-RADS 2018, a lesion classified as probable HCC in KLCANCC 2018 criteria requires at least one item from each of the two categories of ancillary imaging features (favoring malignancy in general and favoring HCC in particular). This difference may contribute to a statistically no significant difference in sensitivity but significantly higher specificity in definite/probable HCC of KLCANCC 2018 as compared with LR-5/4 of LI-RADS 2018. In the present study, LR-5/4 resulted in an unacceptably low specificity, which is consistent with some recent studies and a recent metaanalysis. ${ }^{17-19}$ Meanwhile, definite/probable HCC of KLCA-NCC 2018 maintained high specificity (>90\%). In definite/probable HCC of KLCA-NCC 2018, false positive results were mostly due to CHCC-CCA, defined as primary liver carcinoma with both hepatocytic and cholangiocytic differentiation. ${ }^{20}$ Several studies have also reported that some CHCC-CCAs might not be distinguishable from HCCs based on imaging features. ${ }^{21,22}$

Our study has strength by comparing the diagnostic systems for the noninvasive diagnosis of HCC and identifying proper imaging criteria according to MRI contrast agents. This study indicates that KLCA-NCC 2018 may be more appropriate diagnostic criteria for HCC when using HBA-MRI. A recent prospective study comparing ECA-MRI and HBA-MRI intraindividually for the diagnosis of HCC has shown that the current LR- 5 criteria of LI-RADS limited the sensitivity on HBA-MRI. ${ }^{23}$ In our study, LR-5 of LI-RADS 2018 also revealed suboptimal sensitivity on HBA-MRI, whereas definite HCC of KLCA-NCC 2018 showed higher sensitivity when using HBA-MRI. In particular, there was a greater difference in the sensitivities of $<10-19 \mathrm{~mm}$ lesions on HBA-MRI between LI-RADS and the KLCA-NCC 2018 guidelines. The diagnostic criteria for HCC need to be adjusted according to MRI contrast agent. In addition, imaging criteria reflect geographic differences in clinical environment and treatment strategies. In Korea, early treatment with locoregional therapies is widely used for HCC, thus, the use of KLCA-NCC 2018 diagnostic criteria may provide better sensitivity without compromise in specificity and help detection and early diagnosis of small HCC when using HBA-MRI. ${ }^{5}$

This study has several limitations. First, the retrospective study design at a single center may have introduced an inevitable selection bias. Second, the predominance of patients with chronic hepatitis B in our study population may limit the generalizability of 
the results to other geographic populations with different etiologies of HCC. Additional prospective multicenter studies that include patients with various etiologies of liver disease are required to validate our results. Third, we used the reference standard for the final diagnosis of benign lesion not based on a pathological diagnosis alone but on a composite clinical reference standard. However, pathologic confirmation for highly suspected benign lesions is not recommended in clinical practice, and application of a strict standard of reference (only pathology) for benign lesions may have resulted in a confirmation bias. Finally, the blinded readers had participated in imaging diagnoses in their daily practice; thus, recall bias might have occurred.

In conclusion, LI-RADS 2018 and KLCA-NCC 2018 show comparable diagnostic performances on ECA-MRI. On HBA-MRI, definite HCC of KLCA-NCC 2018 provides better sensitivity and accuracy than LR-5 category of LI-RADS 2018 and definite/probable HCC of KLCA-NCC 2018 reveals higher specificity than LR-5/4 of LIRADS 2018 for the noninvasive diagnosis of HCC.

\section{Authors' contribution}

All authors contributed to the interpretation of results, critical revision of the manuscript, and approved the final manuscript.

-Guarantor of the integrity of the entire study: M-J. Kim

-Study concept and design: S. Lee, S-S. Kim, and M-J. Kim

-Acquisition of data: S. Lee, S-S. Kim, D.R. Chang, and M-J. Kim

-Statistical analysis: H. Kim

-Drafting of the manuscript: S. Lee and M-J. Kim

\section{Acknowledgements}

This study was supported by a grant from the National R\&D Program for Cancer Control, Ministry of Health \& Welfare, Korea (Grant No. 1520160). The funders had no role in the study design, data collection and analysis, decision to publish, or preparation of the manuscript.

\section{Conflicts of Interest}

The authors have no conflicts to disclose.

\section{SUPPLEMENTARY MATERIAL}

Supplementary material is available at Clinical and Molecular Hepatology website (http://www.e-cmh.org).

\section{REFERENCES}

1. Asrani SK, Devarbhavi H, Eaton J, Kamath PS. Burden of liver diseases in the world. J Hepatol 2019;70:151-171.

2. Marrero JA, Kulik LM, Sirlin CB, Zhu AX, Finn RS, Abecassis MM, et al. Diagnosis, Staging, and Management of Hepatocellular Carcinoma: 2018 Practice Guidance by the American Association for the Study of Liver Diseases. Hepatology 2018;68:723-750.

3. European Association for the Study of the Liver. EASL clinical practice guidelines: management of hepatocellular carcinoma. J Hepatol 2018;69:182-236.

4. Tang A, Cruite I, Mitchell DG, Sirlin CB. Hepatocellular carcinoma imaging systems: why they exist, how they have evolved, and how they differ. Abdom Radiol (NY) 2018;43:3-12.

5. Kim TH, Kim SY, Tang A, Lee JM. Comparison of international guidelines for noninvasive diagnosis of hepatocellular carcinoma: 2018 update. Clin Mol Hepatol 2019;25:245-263.

6. American College of Radiology (ACR). CT/MRI LI-RADS $®$ V2018. ACR web site, <https://www.acr.org/Clinical-Resources/Reportingand-Data-Systems/LI-RADS/CT-MRI-LI-RADS-v2018>. Accessed 8 Aug 2018.

7. Park JW; Korean Liver Cancer Study Group and National Cancer Center. Practice guideline for diagnosis and treatment of hepatocellular carcinoma. Korean J Hepatol 2004;10:88-98.

8. Korean Liver Cancer Study Group and National Cancer Center, Korea. Practice guidelines for management of hepatocellular carcinoma 2009. Korean J Hepatol 2009;15:391-423.

9. Korean Liver Cancer Study Group (KLCSG); National Cancer Center, Korea (NCC). 2014 KLCSG-NCC Korea practice guideline for the management of hepatocellular carcinoma. Gut Liver 2015;9:267317.

10. Korean Liver Cancer Association; National Cancer Center. 2018 Korean Liver Cancer Association-National Cancer Center Korea practice guidelines for the management of hepatocellular carcinoma. Gut Liver 2019;13:227-299.

11. Korean Liver Cancer Association (KLCA); National Cancer Center (NCC), Goyang, Korea. 2018 Korean Liver Cancer Association-National Cancer Center Korea practice guidelines for the management of hepatocellular carcinoma. Korean J Radiol 2019;20:1042-1113.

12. Choi SH, Byun JH, Lim YS, Yu E, Lee SJ, Kim SY, et al. Diagnostic criteria for hepatocellular carcinoma $\leq 3 \mathrm{~cm}$ with hepatocytespecific contrast-enhanced magnetic resonance imaging. J Hepatol 2016;64:1099-1107.

13. Joo I, Lee JM, Lee DH, Jeon JH, Han JK. Retrospective validation of a new diagnostic criterion for hepatocellular carcinoma on gadoxetic acid-enhanced MRI: can hypointensity on the hepatobiliary phase be used as an alternative to washout with the aid of ancillary features? Eur Radiol 2019;29:1724-1732. 
14. Kim DH, Choi SH, Kim SY, Kim MJ, Lee SS, Byun JH. Gadoxetic acidenhanced MRI of hepatocellular carcinoma: value of washout in transitional and hepatobiliary phases. Radiology 2019;291:651-657.

15. Chernyak V, Fowler KJ, Kamaya A, Kielar AZ, Elsayes KM, Bashir $M R$, et al. Liver Imaging Reporting and Data System (LI-RADS) version 2018: imaging of hepatocellular carcinoma in at-risk patients. Radiology 2018;289:816-830.

16. Fowler KJ, Potretzke TA, Hope TA, Costa EA, Wilson SR. LI-RADS M (LR-M): definite or probable malignancy, not specific for hepatocellular carcinoma. Abdom Radiol (NY) 2018;43:149-157.

17. Kim YY, An C, Kim S, Kim MJ. Diagnostic accuracy of prospective application of the Liver Imaging Reporting and Data System (LI-RADS) in gadoxetate-enhanced MRI. Eur Radiol 2018;28:2038-2046.

18. Ronot M, Fouque O, Esvan M, Lebigot J, Aubé C, Vilgrain V. Comparison of the accuracy of AASLD and LI-RADS criteria for the noninvasive diagnosis of HCC smaller than $3 \mathrm{~cm}$. J Hepatol 2018;68:715723.

19. Kim DH, Choi SH, Park SH, Kim KW, Byun JH, Kim SY, et al. Metaanalysis of the accuracy of Liver Imaging Reporting and Data System category 4 or 5 for diagnosing hepatocellular carcinoma. Gut
2019;68:1719-1721.

20. Brunt E, Aishima S, Clavien PA, Fowler K, Goodman Z, Gores G, et al. CHCC-CCA: consensus terminology for primary liver carcinomas with both hepatocytic and cholangiocytic differentation. Hepatology 2018:68:113-126.

21. Fraum TJ, Tsai R, Rohe E, Ludwig DR, Salter A, Nalbantoglu I, et al. Differentiation of hepatocellular carcinoma from other hepatic malignancies in patients at risk: diagnostic performance of the Liver Imaging Reporting and Data System version 2014. Radiology 2018;286:158-172.

22. Jeon SK, Joo I, Lee DH, Lee SM, Kang HJ, Lee KB, et al. Combined hepatocellular cholangiocarcinoma: LI-RADS v2017 categorisation for differential diagnosis and prognostication on gadoxetic acidenhanced MR imaging. Eur Radiol 2019;29:373-382.

23. Min JH, Kim JM, Kim YK, Kang TW, Lee SJ, Choi GS, et al. Prospective intraindividual comparison of magnetic resonance imaging with gadoxetic acid and extracellular contrast for diagnosis of hepatocellular carcinomas using the Liver Imaging Reporting and Data System. Hepatology 2018;68:2254-2266. 